2. Do Loe, Greenhill - Principios y práctica de obstetricia. VIII edición. Cap. LXXII. Parto provocado.

3. W. Stoockel - Tratado de obstetricia. III Ed. espiñola. Embarazo y parto múltiples.

4. De Lee, Greenhill - Embarazo múltiple. Cap. XXXIV.

5. W. Streckel - Anomalías de desarrollo del feto.

6. Ramírez Merchón - Conductas obstétricas controvertibles. Revista de la Facultad de Medicina. Uni- versidad Nacional. Numeros / y 0 de 1948. Vol. XVI.

7. Silva Molica - Comentarios clíni$\cos$ y estadísticos sobre operación cesárea. Trabajo inédito leído en marzo de 1950; en la Sociedad Co lombiana de Obstetricia y de Gi. necología.

8. Modina Corvantes - Anotaciones sobre Deontologici en Ginecologia y Obstetricia. Tesis de grado. 1948. Cap. VI. Operaciones ciertamente prohibidas.

\title{
Quinta Asamblea de Estudios Post-graduados en Fndocrinología y Metabolismo
}

La Quinta Asamblea Anual de Estudios Post-graduacios en Endocrinolo. gía y Metabolismo, organizada bajo los ausplcios de "The Endoctine Socle ty" (previamente "Association" for the Study of Internal Secrétions"), se celebrará en Miami Beach, Florida, E. U. de A.; los dias del 3 al 8 inclusive $d$. noviembre próximo-venidero.

En vista de la importancia creciente de los recientes descubrimientos en Endocrinologia y Metabolismo para to dos los campos de la medicina mo

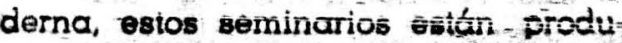
ciendo un grandísimo interés entre lós médicos de nuestro hemisterio. La ma. trícula de inseripción, al principlo jimitada a 100 médicos, ha tentdo que ser ampliada a 200 para acomodar a los que se desean inscribir cada año en mayor número, procedientes de todós los países de-América.
Este año, por primera vez, se le ha de hacer más fácil a los médicos de Latino América el obtener un mayor beneticlo de su asistencia al curso pues se les proveerá la traducelón a. mulitenea al espanol de todas las contorenclas, así como do las preguntas y respuestas, a traves de couriculares ra. diofónicos. Las traducciones serán hechas al micrótono por médicos graduados de universidades latinoamerica. nas que están haciendo internados $y$ residencias en los hospitales de Miami y entrenándose especicimente para capacitarios como traductores rápidtos competentes.

La facultad de profesores está com puesta por 20 eminentes clínicos e investlgadores en los campos de Endo. crinología y Metabolismo miembros de las facultades y cuerpos médicos de alqunas de las más importantes es 
vestigación de los Estados Unidos de América y del Canadá y autores de numerosos trabajos óriginales y libros de texto: doctor Willis E. Brown, Pro fesor y Jefe del Departamento de Obstetricia y Ginecología de la Universí dad de Arkansas: doctor John S. L. Browne. Prolesor de Medicina de la Universidad de MCGill y Director de las Clínicas del Hosptial Royal Victo ría en Montreal, Canadá; doctcr Frank L. Engel, Profesor Asociado de Medici. na y de Fisiología de la Universidad de Duke, Carolina del Norte: doctor Roberto F. Escamilla. Profesor Clínico Asociado de Medicina, Universidad de California en San Francisco; doctor Peter H. Forsham, Profesor Asociado de Medicina y de Pediatría de la Universidad de California en San Francisco; doctor Robert B. Greenblatt, Profesor de Endocrinología del Colegio Médico de Georgia en Augusta; doctor Carl G. Heller, Profesor Clínico Ásociado de Medicina de la Universidad de Oregón en Portiand; doctor Henry F. Ininofelter, Jy. Instructor do Mediciria de la Escuela de Johns Hopkins en Baltimore; doctor Carlos P. Lamar. Director del Departamento de Endocrinología y Metabolismo del Jackson Memorial Hospital en Miami; doc. tor Charles W. Lloyd, de los Departamentos de Medicina- y de Obstetrícia de la Universidad del Estado de Nueva York en su Centro Médico de Siracusa, Nueva Fork; docior ira I. Náthanson, Profesor Clínico Asociado de Cirugia de la Escuela de Mediciná de Harvard y Cirujano del Hospital Ge. neral de Massachusetts en Boston; doctor Rulon W. Rawson. Director de Investigaciones Clínicas del Instituto Sloan-Kettering, Protesor de Medicino co de Visita del Memorial Center de Nueva York; doctor Edward C. Reitenstoin, Ir.. Director del Instituto y Hespi. tal de Investigaciones Médicas y Pro. fesor de Investigación Médica de lo Universidad de Oklahoma: doctor Edward H. Rynearson, lefe de la Sscción de Endocrinología y Metabolismo de la Mayo Clinic de Rochester, Minnesota $a_{i}$ doctor Robert W. Schneider, del Departamento de Endocrinología y $\mathrm{Me}$ tabolismo de la Cleveland Clinic y Profesor Asociado de Medicina Interna del instituto Educacional Frank E. Bunts en Cleveland; doctor Fred $\boldsymbol{A}$. Simmons, Instructor en Ginecología de la Escuela de Medicina de Harvard y Cirujano Asistente del Hospital General de Massachusetts en Boston; doctor Parul Starr, Profesor y Director del Departamento de Medicina Interna de la Universidad de la California del Sur en Los Angeles y Presidente de The Endocrine Society; doctor Henry H. Turner, Profesor Clínico de Medicl. na de la Universidad de Oklahoma; y el doctor Lawson Whilins. Profesor Asociado de Pediatria del Hospital Johns Hopkins en Baltimore.

El curso será una extensa revisión práctica de los últimos desarrollos en Endocrinología y Metabolismo, con énfasis sobre la relación de estos to mas con la medicina práctica en ge: neral. Constará de 64 conferencias que cubrirán tocáas las fuses dé lós, trostor. nos endoctinos y metabólicos, amplta. mente llustradas con proyecciones de "Iantern slides", varias exhibiclones científicas llustrando métodos de dias. nóstico y tratamiento, demostraciones prácticas de técnicas endocrinológicas de laboratorio, presentaclones do: ca- 
sos clínicos y períodos de preguntas y respuestas al final de cada sesión.

El texto de las conferencias, adecuadamente editado y sumarizado, será impreso con las ilustraciones corres pondientes y se proveerá una copia a cada médico matriculado en el curso. Habrá una versión especial -impresa en español para los médicos latinoa mericanos, si el número de los matri culados fuera suficiente para cubrir su costo.

El programa, a pesar de su considorable extensión, ha sido organizado de-tal manera que habrá tlempo- Hbre para descanso y para paseos o -pesquerias de dia y de noche en el órea metropolitana de Mtami, alrededor de la bellisima bahía de Biscayne y con sus famosas playas, parques de recreo y fabulosos hoteles. Habrá una comida precedida de cocteles el lunes día 3 de noviembre para que todos los médicos matriculados tengan oportunidad de conocerse unos a otros y a los miembros de la facultad de protesores. Los médicos latinoamericomos y sus esposas, junto con los profesores de la facultad y sus esposas, serán invitados del miembro local del comité organizador del curso, doctor Carlos P. La mar y señora para una fiesta de confraternidad médica latinoamericana, incluyendo cocteles y buffet, en la tarde del jueves 6 de noviembré.

El curso se celebrará en el Hotel Roney Plaza que, con sus belíisimos jardines tropicales, extensa playa privada, gran piscina de natación, cabana club y "golf putting greens", es considerado uno de los grandes hoteles de playa más famosos del mundo y está completamente alre-acondiclo. nado. Precios especiales de conven. alón han sido concedidos para los miembros de esta asamblea y sus tamiliares que les acompañen. El "Surf Room" en el que se celebrarán las sesiones, está deliciosamente aire-acon dicionado y poseé lluminación - especial que permite tomar notas $y$ ver proyecciones a un mismo tiempo, de un modo confortable.

En la semana qué sigue a la de esta asamblea, se celebrará lambén en Miami la convención anual de la "Southern Medical Association", Ia segunda agrupadión médica del mundo en el número de sus miembros y a la que todos los médicos de Latinoamérica están cordialmente invitados. Unos 10.000 médicos de Norte, Centro y Sur América atenderán esta convención con sus grandes exhlbiciones científicas y técnicas, sesiones plenarias y de especialidades en todas las ramas de la medicina.

La coincidencia especial de la celebración de estos dos importantes eventos científicos en semanas consecut:vas, ofrece una oportunidad única para los médicos en ejercicio práctico y para los que están támbién dedicados a la enseñanza, de ponerse al día en los grandes adelantos de la medicina contemporánea y de gozar con sus famillares de unas placenteras vacaciones en el clima ideal de Miami, a un costo muy por debajo de los precios usuales.

Los interesados dóbeñ escribir directamente $\alpha$ :

Doctor Henry H. Turner, Secretary. Treasurer.

The Endocrine Society.

1.200 North Walker

Oklahoma City 3, Oklahoma, U. 
rara garantuzar la reservacion de un puesto en la asamblea, cada carta de aplicación debe ser acompañada ta giro postal o bancario internacio nal por la cantidad de $\$ 75,00$ U. S. A., cossto de la cuota de matrícula. Al mis. mo tiempo debe escribirse directamen. te al "Hotel Ronel-Plazz: Miami Beach, Florida, U. S. A.", pidiendo las reser. vaciones que se deseen $y$ notificando al hotel que se va a atender esta asamblea, para que se le apliquen los precios especiales convenidos. " La lis te de matrícula se cerrará el 10 de sctubre de -1952. Las aplicaciones se rón aceptadas en el orden que se re ciban hasta el número total de 200.

\section{Ouinta Aeamblea do Estudios Pest- graduados on Endocrinologia y Motabolismo}

\section{Procrand}

Lunes, novtombre 3 de 1952.

8:00-9:00-Inscripción.

Sestón de la Mañana.

Principios y Procodimionios Gonorala.

0.00-9:45-Principio Generales de la Endocrinologia, doctor Starr.

(9) $\$ 10.00, \& 12.00$ y $\$ 14.00$ (U. S. 16 larea) por dia, por cuarto doble, ocupado por una 0 dos personas. (El precio corsesponde at tamatio y posicion del cuarto pero es ol mismo at es una o dos personas que lo coupen). Todos esthn alre-econdiclosades.
9:45 10:30-Intertiolaciones Genora les de las Glándulas Endoctinas, doc. tor Forsham.

- 10:30-10:45-Relaciones Neuro-Hipo Zisarias, doctor Lloyd.

10:45-11:00 Intermedio Retrescos.

11:00-12:00-Control Endoctino del Metabolismo, doctor Engel.

12:00:12:45-Introducción Diagnósti. ca a los problemas endocrinos; evalua ción de las ayudas de laboratorio en el diagnóstico, doctor Escamilla.

\section{Seatón del Modiodía.}

2:00-2:30-Abusos de terapia hormonal, doctor Rynearson.

2:30-3:00-Periodo para preguntas y respuestas, moderador: doctor Starr.

\section{Pitultaria Anterior.}

3:00-3:15-Química y tisiologia del lóbulo pitultario anterlor, doctor Klino felter.

3:15-4:00-Síndromes de hipofunción pituitaria anterior: Panhipopitultarismo, caquexia de simmonds, síndrome de Sheehan, anorexia nervosa, sindro me de Frohlich, doctor Escamilla.

4:00-4:15-Intermedio - Refrescos.

4:15-4:45-Sindromes de -hiperfunción pituitaric anterior: gigantismo $y$ acromegalia, doctor Schneider.

4:45.5:00 Otros síndromes del lóbu. lo anterior: tumores pitultarios, doctor Klinefelter.

5:00-5:30_Periodo para pregunies y respuestas, moderador: doctor Escam! Hla.

6:30-Reunión social - Cocteles y co. mido. 
Martes, noviembre 4 de 1952.

Sesión de la Mañana.

Corteza Suprarrenal.

9:00-9:30-Química y fisiología de la corteza suprarrenal, doctor Engel.

9:30-10:00-Síndrome general de adaptación; enfermedades de adapta. ción, doctor Browne.

10:00-11:00-Síndromes de hipofun. ción adrenocortical: enfermedad de Addison e insuficiencia adreno-cortical de origen pltuitario, doctor Forsham.

11:00-11:15-Intermedio - Refrescos.

11:15-12:00-Sindromes de hiperfunción adreno-cortical: A. Síndrome y entermedad de Cushing, doctor Browne.

12:00-12:45-Síndromes de Hiperfunción adrenc-cortical: B. Síndrome adreno-genital y otros síndromes relacionados, doctor Wilkins.

\section{Sesión del Mediodía.}

2:00-2:45-ACTH y cortisona en tras tornos no endocrinos: artritis, enfermedádes colágenas, etc., doctor Browne.

\section{Médula Suprarrenal.}

2:45-3:15-Química y fisiología de la médula suprarrenal, síndromes adre no-medulares: feocromocitoma, doctor Schneider.

3:15-3:45-Las suprarrenaies y ciertos trastornos no endocrinos: adrenales - hipertensión; adrenalectomía como fratamiento, doctor Forsham.

3:45-4:00-Intermedio - Refrescos.

4:00-4:30--Período para preguntas y respuestas, moderador: doctor Fors ham.
4:30-5:30-Presentacion de casos c11nicos. Trastornos pituitarios y suprarenales, moderador: doctor Lamar.

\section{Noche Libre.}

Miércoles, noviembre 5 de 1952.

\section{Sosib́n de la Matena.}

\section{Pánereas.}

9:00-9:30-Química y fisiología del páncreas; acción fisiológica de la insulinc; lipocaic, doctor Lamar.

9:30-10:00-Síndrome de hipotunción pancreática: diabetes sacarina: A. Etialogia, signos y síntomas, hallazgos de laboratorio, diagnóstico y pronóstico, doctor Escamilla.

10:00-10:30-Síndrome de hipotun. ción pancrétitica: diabetes sacarina: B. Tratamiento del estado agudo: acidosis diabética, doctor Schneider.

10:30-11:00-Síndrome de hipotun. ción pancreática: diabetes sacarina: C. Tratamiento del estado crónico: die. tơ $\theta$ insulina, doctor Lamar.

\section{1:00-11:15-Intermedio - Refrescos.}

11:15-11:30-Síndrome de hipotun. ción pancreáticar diabetes sacarina: D. Problemas insulinicos: reacción o coma y su tratamiento, sensibllidad, resistencia, clergia, lipodistrotias, doctor Rynearson

11:30-12:15-Síndrome de Hipofunción pancreática: diabetes sacarina: E. Complicaciones y situaciones espeelales, complicaciones vasculares, re. 
tinopatia, neuropatia, nefropatia; infancia, gestación, cirugia, infecciones. edad avanzada, doctor Sćhneider.

12:15-12:45-Síndrome de hipofunción pancreática:- diabetes sacarina: F. Diabetes como complicación de otros trastorros endocrinos. doctor Rey. nectson.

12:45-1:15--Sindrome de hipertunción pancreática: tumores de las islas de Langerhans: hipoglicemia funcio nal, doctor Rynearson.

\section{Modio Dí Libro. \\ Soulón do la Nocho.}

7:30-7:45-Insulina en trastornos no endoerinos; doctor Escamilla.

7:45-8:00-Factores Endocrinos no pancroáticos en el metabolismo de los carbohidratos, doctor Engel.

8:00-8:30-Periodo para preguntas y respuestas, moderaidor: doctor Lamar.

\section{Nutrielon.}

8:00-8:45-Interrelaciones de las hormonas, vitaminas y nutrición doctor Lamar.

8:45-9:15-Obesidad Y delgadez, doctor Rynearson.

\section{Philtaria poatector.}

9:15-10:00-Quimica, fisiología o interrelaciones neurohipotisarias de la pltuliaria posterior: ándromes posteropituitarlos, doctor Lloyd. lueves, noviombre 6 do 1952.

\section{Sealós do la Metiana.}

\section{Troldes.}

9:00-9:30-Química y fisiologia de la tiroides doctor Rawson.

9:3010:15-Sindromes de hipotunción tiroldea: hipotiroidismo y mixedema, doctor Starr.

10:15-11:00-Sindromes de hipertunción tiroidecs hipertiroidismo, tirotoxicosls y enfermedad de Graves-Basedow, doctor Rawson.

11:00-11:15-Intermedio - Refrescos.

11:15-12:00-Otros sindromes tiroideos: bocio notóxico, cóncer tiroidj. tis, doctor Rawson.

12:00-12:15-Hormona Tiroidea en trastornos noendocrinos, doctor Star.

12:15-12:45-Periodo para pregun. tas $y$-respuestas moderador: doctor Rawson.

\section{Seatón dol Madiodía.}

\section{Adolesconcla - Infancia.}

2:00-2:30-Fisiología de la adoles. cencia; variaciones constituclonales en la norma; pubertad precoz y demo rada; prematurez, doctor Wilkins.

2:30-3:15-Foctores s, ondocrinos $Y$ otros que Influenatan- ol crecimiento comático y el decartollo; sindrames ondoctinos en la infancia: A. Enanis. mo, doctor Wilktns.

3:15-3:30-sindromes endocinios on la intancia: B. Hipotiraldinmo, doctor Willedins. 
Sábado, noviembre 8 de 1952.

\section{Sesión de la Mañana.}

\section{Testículo8.}

9:00-9:15-Quimica y fislología de los testículos, doctor Heller.

9:15-9:45-Sindromes de hipofuncion testicular: A Sindromes hipergonado. trólicos: eunucos, eunucoides, climaterio masculino, doctor Heller.

9:45-10:00-Síndromes de hipofunción testicular: A. Síndromes hipergo. nadotróficos: sindrome de Klinefelter. doctor Klinefelter.

10:00-10:30-Sindromes de hipotunción testiculcr: B. Síndromes hipogonadotróficos: eunucoides, insuficiencia testicular secundaria a insuficiencia pituitaria, doctor Heller.

10:30-10:45-Síndromes de Hiperfunción testicular y tumores: de células de Leydig, corionepitelioma y otros, doc. tor Simmons.

10:45-11:15-Aspectos endocrinós de trastornos de la mama en el hombre: ginecomastia; mamoplasic de la adalescencia, doctor Klinefelter.

11:15-11:30-Intermedio - Retrescos,

11:30-12:00 Esterilidad en el hom. bre, doctor Simmons.

12:00-12:30-Hormonas sexuales on trastornos noendocrinos en el hombre: cáncer de la próstata y de otrós órganos, convalecencia, doctor Na. thaneon.

12:30-12:45-Criptorquidismo, doc tor Heller.

\section{Sesión del Mediodía.}

2:00-2:30-Periodo pára preguntas y respuestas, moderador: doctor Klinefelter.

\section{Paratiroides.}

2:30.2:45-Química y tis ologia de las paratiroides, doctor Rejfenstein

2:45-3:15-Sindromes paratiroides: hipo e hiperparatiroldismo, doctor Rei. fenstein.

3:15-3:45-Entermedades metabólicas de los huesos; sus relaciones e: las glándulas endocrinas: osteitis tibrosa generalizada, osteomalacia, osteoporosis, doctor Reifenstein.

3:45-4:00-Intermedio - Retrescos.

4:00-4:30-Periodo para preguntas y respuestas, moderador: doctor Reifenstein.

4:30-5:30-Presentación dé casos clí nicos: trastornos gonctales y paratiroldeos, moderador: docfor Lamar.

Fin de la Asamblea.

N. de la R.-No parece diticil que la Sociedad Colombiana de Endocrinologia y la de Obstetricia y Ginecolo. gía pagaran la matrícula a uno de Iu tuviórcimos esta representación on el Congreso de Miaml. 
3:30-4:00-Período para preguntas y respuestas, moderador: doctor Wilkins.

4:00-4:15-Intermedio - Refrescos.

\section{Miscelánea.}

4:15-4:30-Pseudo - endocinopatias doctor Turner.

4:30-5:30-Piesentación de casos cli nicos: trastornos pancreáticos y tiroi déos moderador: doctor Lamar.

\section{Noche Libre.}

7:00-Cocteles y buffet en honor de los miembros de la Facultad y médi. cos latinoamericanos, con sus señoras, ofrecido por el doctor Lamar y señora.

\section{Vierneg, noviembre 7 de 1952.}

Sesión de la Mañana.

\section{Ovarios.}

9:00-9:30-Química y fisiologia de los ovarios: fisiología de la menstruacion, doctor Lloyd.

9:30-10:00-Sindromes de hípotunción ovárica: A. Sindromes hipergonadotróficos: castración, menopausia; hi. pogonadismo, doctor Brown.

10:00-10:15 - Sindromes de hipolua ción ovárica: A. Síndromes hipergonadotróficos: agenesia ovárica y sindro me de-Turner, doctor Turner.

10:15-11:00-Sindromes de hipofun. ción ovariea: B. Sindromes hipogonadotróficos: hipogonadismo, insuficien: cla ovárica secundaria a insufictencia pituitaria, doctor Greenblatt.
11:00-11:15-Intermedio - Refrescos.

11:15-11:45_Sindromes de hipofunción ovárica: C. Sindromes normogonadotróficos: metropatia hemorrágica; dismenorrea, endometriosis, doctor Greenblatt.

11:45-12:00-Sindromes de hipofunción ovárica: C. Sindromes normogo ncidotróticos: amenorrea hipotalámica doctor Relfenstein.

12:00-12:15-Sindromes de hiperfun. ción ovárica y tumores: de células gra. nulosas, de células de la ceca, Luteoma y otros, doctor Greenblatt.

12:15-12:45-Aspectos endocrinos de trastornos benignos de la mama en la muier, doctor Nathanson.

12:45-1:15-Período para preguntas y respuestats, moderador: doctor Turner.

\section{Medio Día Libre.}

\section{Seatión de la Noche.}

\section{Placenta.}

7:30-7:45-Química y-fisiología de la placenta, doctor Brown.

7:45-8:30-Fisiología de la gestación: aspectos endocrinos de trastornos de la gestación: aborto habitual, toxemic's de la gestación, gestación en diabetes, corionepitelioma, mola hidatidiforme, etc., doctor Brown.

8:30-9:00-Esterilidad en la mujer, doctor Simmons.

9:00-9:30-Hormonas sexuales en trastornos no-endocrinos de la mujer: cáncer de la mama y de otros órga. nos, convalecencia, doctor Nathan: son.

9:30-10:00-Período para preguntas y respuestas, moderador doctor Brown. 